\title{
Optical-field-controlled photoemission from plasmonic nanoparticles
}

\author{
William P. Putnam ${ }^{1,2 \star}$, Richard G. Hobbs ${ }^{2,3}$, Phillip D. Keathley ${ }^{2}$, Karl K. Berggren ${ }^{2}$ \\ and Franz X. Kärtner ${ }^{1,2,4}$
}

\begin{abstract}
At high intensities, light-matter interactions are controlled by the electric field of the exciting light. For instance, when an intense laser pulse interacts with an atomic gas, individual cycles of the incident electric field ionize gas atoms and steer the resulting attosecond-duration electrical wavepackets ${ }^{1,2}$. Such field-controlled light-matter interactions form the basis of attosecond science and have recently expanded from gases to solid-state nanostructures ${ }^{3-18}$. Here, we extend these field-controlled interactions to metallic nanoparticles supporting localized surface plasmon resonances. We demonstrate strong-field, carrier-envelope-phase-sensitive photoemission from arrays of tailored metallic nanoparticles, and we show the influence of the nanoparticle geometry and the plasmon resonance on the phase-sensitive response. Additionally, from a technological standpoint, we push strong-field light-matter interactions to the chip scale. We integrate our plasmonic nanoparticles and experimental geometry in compact, microoptoelectronic devices that operate out of vacuum and under ambient conditions.
\end{abstract}

Moving from low to high optical intensity, photoemission goes from photon-driven to field-controlled. Consider illuminating a metallic surface with an infrared femtosecond laser pulse with electric field $F(t)=F_{0} A(t) \cos (\omega t+\varphi)$, where $F_{0}$ is the peak field, $A(t)$ is the normalized pulse envelope, $\omega$ is the carrier frequency, and $\varphi$ is the carrier-envelope phase (CEP). When the pulse interacts with the metallic surface, electrons are excited out of the metal and into the surrounding vacuum. At typical incident intensities, this photoemission process is photon-driven: emission is dictated by the pulse's photon energy, that is, $\hbar \omega$, and photon flux, that is, the pulse's intensity envelope $\propto\left|F_{0} A(t)\right|^{2}$. At high intensities, this photoemission process resembles field-controlled tunnelling. The strong electric field of the pulse deflects the binding potential of the metallic surface and drives electron tunnelling through the distorted barrier. This tunnelling occurs over a timescale $\tau_{t}=\sqrt{2 m W_{\mathrm{F}}} / e F_{0}$, where $W_{\mathrm{F}}$ is the workfunction of the surface, $m$ is the electron mass, and $e$ is its charge ${ }^{19,20}$. With sufficiently strong $F_{0}, \tau_{t}$ becomes shorter than the characteristic cycle time of the exciting laser light $\left(\tau_{t}<\tau_{\text {cyc }}=1 / \omega\right)$, and individual cycles of the driving electric field eject subcycle electrical bursts from the metal and steer these ultrafast currents through the surrounding vacuum ${ }^{6,8}$; in this strongfield regime, photoemission is controlled by the driving optical electric field and, accordingly, by the CEP, $\varphi$.

In recent years, metallic nanotips have emerged as platforms to non-destructively investigate photoemission in the strong-field regime. When a nanotip is illuminated by a femtosecond laser pulse, the incident field is locally enhanced at the apex of the tip. Due primarily to the tip's sharp geometry, the field enhancement is typically $<10$, and the temporal profile of the enhanced field, $F_{\text {tip }}(t)$, approximately follows that of the instantaneous incident field ${ }^{21,22}$. With typical incident intensities, $F_{\text {tip }}(t)$ can drive strong-field processes: photoemission current yields and photoelectron energy spectra from nanotips have shown strong-field characteristics ${ }^{3-5,7,10,11,14,15}$, and exciting nanotips with phase-stabilized laser pulses, CEP-sensitive signatures have been observed ${ }^{5,14}$.

Compared with nanotips, metallic nanoparticles offer higher field enhancements as well as additional resonant and geometric degrees of freedom. When a laser pulse illuminates a metallic nanoparticle, the incident field can resonantly drive collective oscillations of the particle's conduction electrons ${ }^{23,24}$. These localized surface plasmon resonances yield dramatically enhanced local fields, and similar to nanotips, metallic nanoparticles have shown strong-field behaviours in their photoemission currents and energy spectra ${ }^{9,12,13,16-18}$.

Here, we report the first measurements of CEP-sensitive, strongfield photoemission from metallic nanoparticles. We integrate arrays of metallic nanoparticles into micro-optoelectronic devices, and illuminating the nanoparticles with near-infrared laser pulses, we observe large photoemission currents up to $\approx 30 \mathrm{nA}$ with CEP-sensitive modulations up to $\approx 1.5 \mathrm{pA}$ (all under ambient conditions). Investigating resonant and off-resonant arrays of rodand triangle-shaped particles, we explore the influence of the nanoparticles' resonant and geometric degrees of freedom on the CEP-sensitive response, and the results are well explained by a quasistatic tunnelling model. From a practical, technological standpoint, our measurements are informative for designs of future ultrafast, ultrabright nanostructured photocathodes, and our devices could provide a route towards chip-scale, CEP-sensitive photodetectors. From a more general perspective, this work takes a step towards pushing optical-field-controlled, attosecond science and technology from elaborate laboratory settings to on-chip devices operating in ambient conditions.

In Fig. 1 we illustrate our experimental geometry. Figure 1a shows an example device: the device resembles a microscale phototube, that is, a vacuum tube with an optically excited emitter or photocathode. The emitter consists of an array of gold nanoparticles resting on a 60-nm-thick layer of transparent, conducting indium tin oxide (ITO) and separated from an ITO collector, or anode, by a $\approx 5 \mu \mathrm{m}$ gap. In Fig. 1b, we provide an optical microscope image of one

\footnotetext{
${ }^{1}$ Department of Physics and Center for Ultrafast Imaging, University of Hamburg, Luruper Chaussee 149, 22761 Hamburg, Germany. ${ }^{2}$ Department of Electrical Engineering and Computer Science and Research Laboratory of Electronics, Massachusetts Institute of Technology, 77 Massachusetts Avenue, Cambridge, Massachusetts 02139, USA. ${ }^{3}$ Centre for Research on Adaptive Nanostructures and Nanodevices (CRANN), Advanced Materials and Bio-Engineering Research Centre (AMBER), and School of Chemistry, Trinity College Dublin, Dublin 2, Ireland. ${ }^{4}$ Center for Free-Electron Laser Science

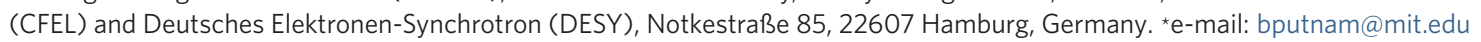




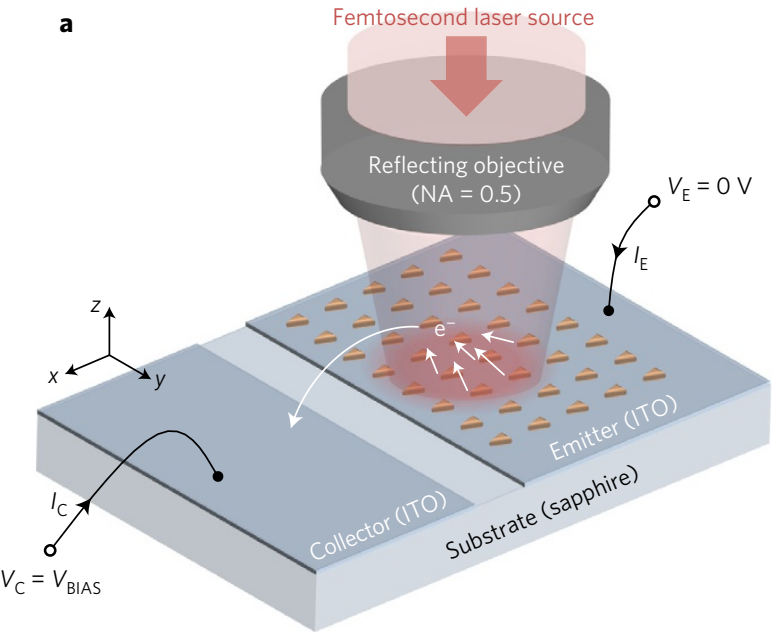

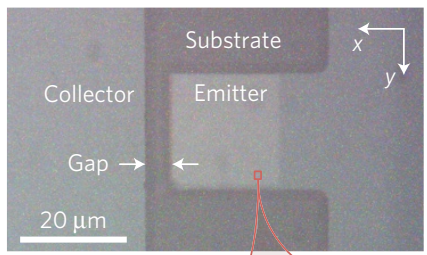

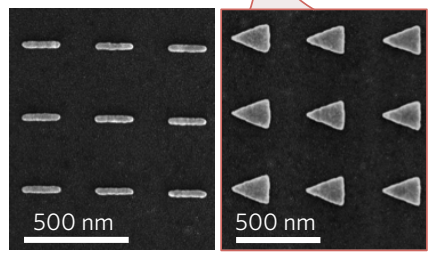

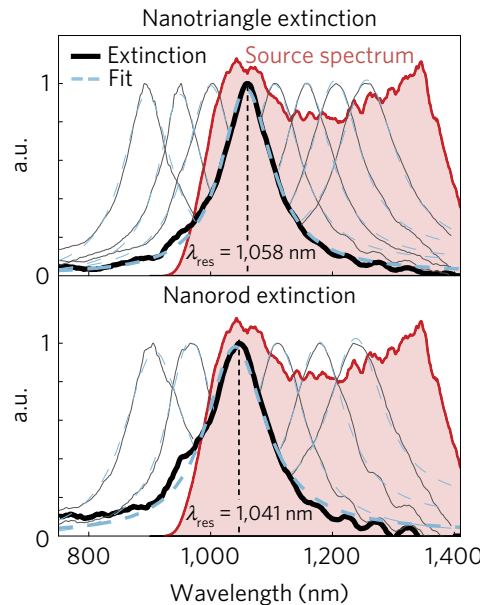

Figure 1 | Device layout and basic operation. a, Sketch of example device and experimental arrangement. An array of nanoparticle emitters is illuminated by tightly focused, femtosecond laser pulses (the device sits out of vacuum in ambient laboratory conditions). The illuminated nanoparticles emit electrons that are swept by a positive bias voltage $\left(V_{B I A S}\right)$ from emitter to collector. $\mathbf{b}$, (Top) Optical microscope image of an emitter array. The dark region is the sapphire substrate, and the collector and emitter electrodes are labelled. The light square is a $20 \mu \mathrm{m} \times 20 \mu \mathrm{m}$ array of nanotriangle emitters. (Bottom) Scanning electron micrographs of example nanotriangle and nanorod arrays (the red box bounding the nanotriangle micrograph corresponds to the red box in the optical micrograph). c, Measured extinction spectra for the nanotriangle and nanorod arrays (with damped harmonic oscillator fits) and the spectrum of the femtosecond laser source. The prominently displayed (bold) extinction spectra correspond to the arrays displayed in $\mathbf{b}$, and the fits to these spectra yield resonant wavelengths of $\lambda_{\text {res }}=1,058 \mathrm{~nm}$ and $1,041 \mathrm{~nm}$ and quality factors of $Q=10.3$ and 8.7 for the nanotriangle and nanorod arrays respectively.

of our devices and electron micrographs of example nanorod and nanotriangle emitters. In our experiments, we investigate fourteen different nanoparticle emitter devices: eight arrays of differently sized nanotriangles and six arrays of differently sized nanorods. The measured optical extinction spectra for each array are plotted in Fig. 1c and show localized surface plasmon resonances with resonant wavelengths, $\lambda_{\text {res }}$, ranging from $895 \mathrm{~nm}$ to $1,256 \mathrm{~nm}$. Further device fabrication and characterization details are provided in Supplementary Section I.

In our experiments we illuminate our nanoparticle emitters with femtosecond laser pulses. With a repetition rate of $f_{\mathrm{R}}=78 \mathrm{MHz}$, these femtosecond pulses have a $\cos ^{2}$-shaped envelope with a fullwidth at half-maximum duration of $10 \mathrm{fs}$, a tunable pulse energy, $E_{\mathrm{P}}$, up to $200 \mathrm{pJ}$, and a centre wavelength of $\lambda=1,177 \mathrm{~nm}$ (further laser source details are provided in Supplementary Section II). The femtosecond pulses are tightly focused to an incident peak field of $1.2 \mathrm{~V} \mathrm{~nm}^{-1}$ (at $E_{\mathrm{P}}=200 \mathrm{pJ}$ ) and drive large photoemission currents from the nanoparticles. These currents are pulled across the emitter-collector gap by a positive bias voltage applied to the collector, $V_{\mathrm{C}}=V_{\text {BIAS. }}$ ( The bias voltages applied to the emitter and collector are denoted $V_{\mathrm{E}}$ and $V_{\mathrm{C}}$ respectively, and similarly, the currents entering these electrodes are labelled $I_{\mathrm{E}}$ and $I_{\mathrm{C}}$ ). We should note that the mean-free path of few-electronvolt electrons in ambient air is on the order of a few hundred nanometres, and prior demonstrations of field-emitting electronic devices in ambient conditions have typically used emitter-collector gaps below this length scale $\mathrm{e}^{25-28}$. However, in our devices, the photoemission physics occurs within the confined spatial range of each nanoparticle's enhanced local field, that is, within $\sim 10 \mathrm{~nm}$. Additionally, the emitter-collector gap is sufficiently small for the emitted electrons to reliably transit the gap without being captured by the ambient air ${ }^{29}$. Therefore, the device operation is largely unaffected by the ambient conditions. Further discussion regarding ambient operation is included in Supplementary Section III.

In a first experiment, we record the photoemission current as the incident pulse energy is changed. Figure 2 a displays $I_{\mathrm{E}}$, measured at $V_{\text {BIAS }}=30 \mathrm{~V}$, as a function of $E_{\mathrm{p}}$ for the nanotriangle and nanorod arrays shown in Fig. 1b. We fit the low-pulse-energy section of the curve to the conventional multiphoton photoemission form, $I_{\mathrm{MP}} \propto\left|F_{0}\right|^{2 N}$, where $F_{0}$ is the incident peak field. We find $N=5.4$ and 6.1 for the nanorods and nanotriangles respectively (with a photon energy of $\approx 1.1 \mathrm{eV}$ and the workfunction of gold, $W_{\mathrm{F}}=5.1 \mathrm{eV}$, we expect $N \approx 5$ ). In Fig. $2 \mathrm{a}$, we see that as $E_{\mathrm{p}}$ increases, the photoemission curves bend over and depart from the multiphoton form: the emission enters the strong-field regime. (A complete discussion of space-charge limitations and associated measurements and calculations are provided in Supplementary Section IV.)

In the strong-field regime, we expect the emission process to resemble tunnelling. Transition into this regime is marked by the Keldysh (or adiabaticity) parameter, $\gamma=\tau_{t} / \tau_{\mathrm{cyc}}=\omega \sqrt{2 m W_{\mathrm{F}}} / e F_{\mathrm{P}}$, where $F_{\mathrm{P}}$ is the enhanced peak field, that is, $F_{\mathrm{P}}=g F_{0}$, where $g$ is the field enhancement and $F_{0}$ is the incident peak field. In the adiabatic or quasi-static regime, when $\gamma<1$, the photoemission current approximately follows the static field-emission rate driven by the instantaneous value of the enhanced laser field. Here, we take the static field-emission rate to be given by the Fowler-Nordheim form $^{30}, I_{\mathrm{FN}}\left(F_{\text {d.c. }}\right) \propto F_{\text {d.c. }}{ }^{2} \exp \left(4 \sqrt{2 m W_{\mathrm{F}}^{3}} / 3 \hbar e F_{\text {d.c. }}\right)$, where $F_{\text {d.c. }}<0$ is the applied static field $\left(I_{\mathrm{FN}}\left(F_{\mathrm{d} . c .}\right)=0\right.$ for $\left.F_{\text {d.c. }}>0\right)$. Using this form, the emitter current from the nanoparticles in the strong-field regime can be described as:

$$
I_{\mathrm{E}}(\varphi)=f_{\mathrm{R}} \times \int_{-T_{\mathrm{R}} / 2}^{T_{\mathrm{R}} / 2} I_{\mathrm{FN}}\left(F_{\mathrm{np}}(t ; \varphi)\right) \mathrm{d} t
$$

In the above, $T_{\mathrm{R}}=1 / f_{\mathrm{R}}$, and $F_{\mathrm{np}}(t ; \varphi)$ is the localized, enhanced field at the emitting nanoparticles (written here as a function of $\varphi$ ). If we assume that the emission is dominated by the peak of $F_{\mathrm{np}}(t ; \varphi)$, we can approximate equation (1) as $I_{\mathrm{E}} \propto I_{\mathrm{FN}}\left(F_{\mathrm{P}}\right)$, where, as before, $F_{\mathrm{P}}=g F_{0}$ is the enhanced peak field. Fitting this simplified expression to the data in Fig. 2a, we can extract experimental estimates for the field enhancement; we find $g \approx 32$ for both the nanorod and nanotriangle emitter data displayed. Further information concerning this fitting is included in Supplementary Section IV.

The experimentally extracted field enhancements predict large peak fields at the nanoparticles. For the data displayed in Fig. 2a, the predicted peak fields are $\approx 40 \mathrm{~V} \mathrm{~nm}^{-1}$. Previous strong-field 
a
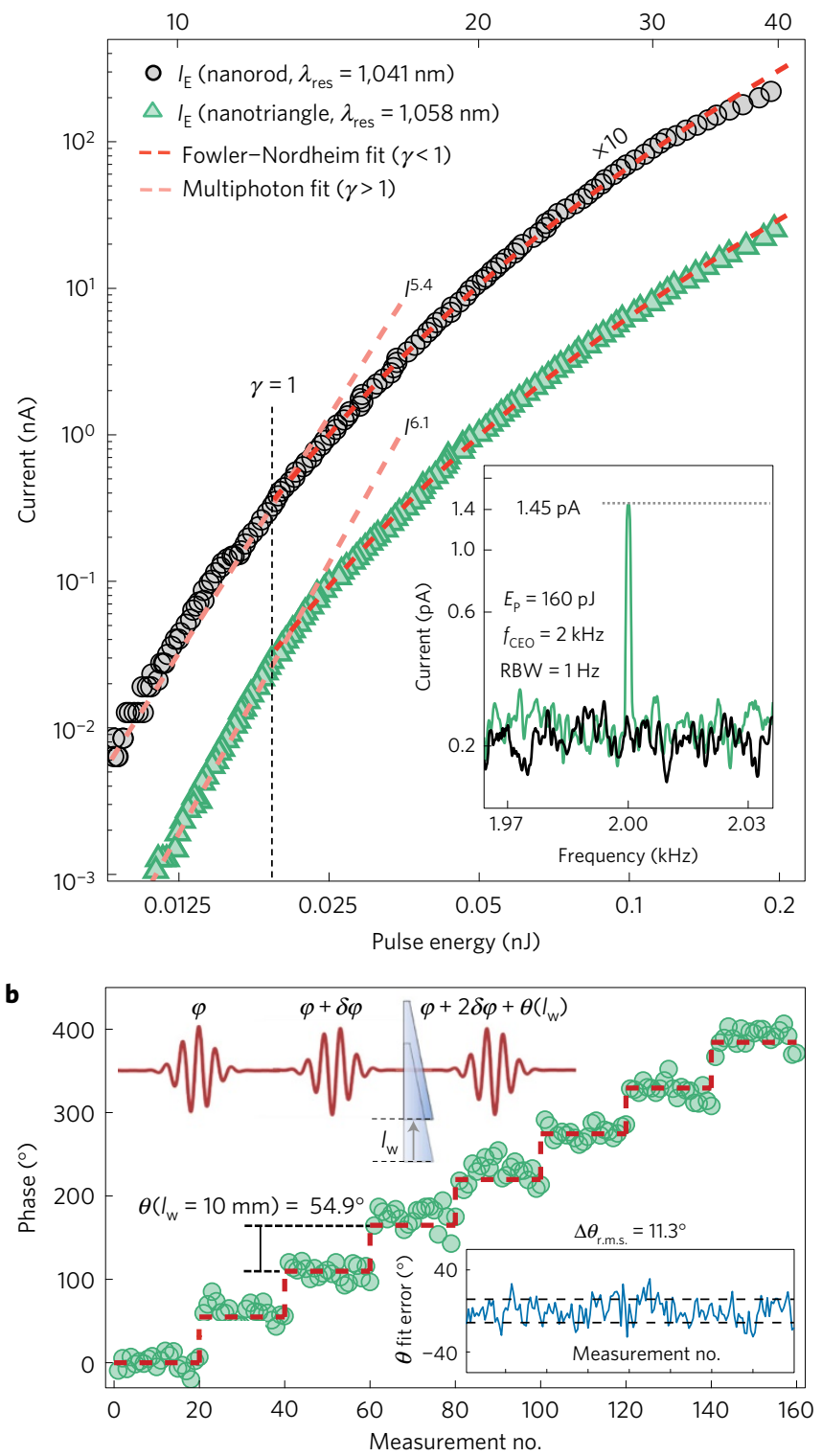

Figure 2 | Strong-field, CEP-sensitive photoemission currents. a, Emitter current versus pulse energy for the nanorod and nanotriangle arrays shown in Fig. $1 \mathrm{~b}$ (nanorod data are shifted up by a factor of 10 for clarity). Fits to the multiphoton and strong-field emission regimes are done self-consistently in the $\gamma<1$ and $\gamma>1$ regions respectively (see Supplementary Section IV for details). The Fowler-Nordheim fits to the strong-field regime predict a field enhancement of $\sim 32$ for both the nanorod and nanotriangle emitters; the $\gamma=1$ point and the peak electric field axis are extrapolated from this field enhancement. The inset shows the spectrum of the emitter current (measured with $E_{\mathrm{p}}=160 \mathrm{pJ}$ ) from the nanorods (black) and nanotriangles (green) with $f_{\mathrm{CEO}}=2 \mathrm{kHz}$ (resolution bandwidth, $\mathrm{RBW}=1 \mathrm{~Hz}$ ). b, Phase response of the $f_{C E O}$ signal from the nanotriangles. A glass wedge is incrementally inserted, and the phase of the signal relative to the local oscillator to which the $f_{C E O}$ of the laser pulse train is stabilized is recorded. The dashed red trace shows a regular staircase fit to the data, and the inset shows the deviation of the measured phase-stepping behaviour from the fit.

investigations with metallic nanoparticles have shown similar extracted field enhancements ${ }^{12,13,17,18}$ and peak fields $s^{9,12,13}$, and these high peak fields could lead to nanoparticle damage via electromigration or field evaporation ${ }^{17,31}$. However, over $10 \mathrm{~min}$ of continuous illumination, we observe current fluctuations of only $\approx 2 \%$, and subsequent inspection of the emitters reveals minimal damage. Further information on device damage is included in Supplementary Section III.

Having demonstrated strong-field operation, we next investigate the CEP-sensitive response of the photoemission. The incident laser pulses are CEP-stabilized: from pulse to pulse, $\varphi$ shifts by a fixed amount $\delta \varphi$, such that $\varphi$ circles $2 \pi$ in phase at the carrierenvelope offset frequency $f_{\mathrm{CEO}}=f_{\mathrm{R}} \times \delta \varphi / 2 \pi=2 \mathrm{kHz}$. In the inset of Fig. $2 \mathrm{a}$, we plot a measurement of the spectrum of the emitter current $I_{\mathrm{E}}(f)$ from the nanotriangle and nanorod arrays in Fig. 1b (green and black curves respectively). The nanotriangle current shows a pronounced modulation at $f_{\mathrm{CEO}}$ with an amplitude of $I_{\mathrm{E}}\left(f=f_{\text {CEO }}\right)=1.45 \mathrm{pA}$ and a signal-to-noise ratio (SNR) of $16 \mathrm{~dB}$ measured with a resolution bandwidth (RBW) of $1 \mathrm{~Hz}$, while the nanorods show no $f_{\text {CEO }}$ response.

To confirm the CEP sensitivity, we investigate the phase response of the $f_{\mathrm{CEO}}$-sensitive signal from the nanotriangles. As illustrated in Fig. 2b, we slide a barium fluoride wedge through the incident laser pulse train. Due to the mismatched group and phase velocities in the glass wedge, the CEP of a pulse traversing the wedge will shift by $\theta$. This phase shift is dependent on the amount of glass traversed and is therefore a function of the wedge insertion; we write $\theta\left(l_{\mathrm{w}}\right)$, where $l_{\mathrm{w}}$ is the length of wedge inserted relative to some reference position. For our laser parameters, we expect that $\theta\left(l_{\mathrm{w}}=10 \mathrm{~mm}\right)=58.3^{\circ}$. Monitoring the phase of the $f_{\mathrm{CEO}}$-sensitive signal relative to the local oscillator that the $f_{\mathrm{CEO}}$ is locked to, we incrementally insert the wedge in steps of $10 \mathrm{~mm}$. In Fig. 2b, we show 160 measurements of $\theta$; every 20 measurements we shift the wedge by an additional $10 \mathrm{~mm}$. We see that the phase increments as expected. Fitting a regular staircase shape to the data, we find that $\theta\left(l_{\mathrm{w}}=10 \mathrm{~mm}\right) \approx 54.9^{\circ} \pm 11.3^{\circ}$, where the $\pm 11.3^{\circ}$ deviation derives from looking at the r.m.s. deviation of the phase from the fit, $\Delta \theta_{\text {r.m.s. }}$ (see inset of Fig. 2b).

Having confirmed CEP sensitivity from the nanotriangles, let us now consider the nanorods. In the strong-field regime, photoemission is dictated not only by the strength, but also by the direction of the driving optical field: emission occurs when the driving optical field bends the binding potential downwards. Consider Fig. 3a; in the strong-field regime, the nanoparticles will only emit when the field points into the gold surface (when the potential is bent downwards), so the nanotriangles, with significant field enhancement only at their apex, emit during the red half-cycles, while the nanorods, with significant field enhancement at both ends, emit during both red and blue half-cycles. From the perspective of a simple one-dimensional emission model such as that presented in equation (1), each nanorod resembles two nanotriangles with their apices pointed in opposite directions. When illuminated by a laser pulse with $\operatorname{CEP} \varphi$, that is, when illuminated with field $F(t, \varphi)$, a nanorod's photoemission response equals the sum of a nanotriangle's response to $F(t, \varphi)$ and $-F(t, \varphi)$. Noting that $F(t, \varphi+\pi)=-F(t, \varphi)$, we can write $I_{\mathrm{E}}^{\text {rod }}(\varphi)=I_{\mathrm{E}}^{\text {tri }}(\varphi)+I_{\mathrm{E}}^{\text {tri }}(\varphi+\pi)$, where $I_{\mathrm{E}}^{\text {rod }}$ and $I_{\mathrm{E}}^{\text {tri }}$ are the emitter currents from the nanorods and nanotriangles respectively. Since $I_{\mathrm{E}}^{\text {tri }}(\varphi)$ is periodic with period $2 \pi$ (the field is periodic in $\varphi$ with period $2 \pi$ ), we see that $I_{\mathrm{E}}^{\text {rod }}(\varphi)$ is periodic with period $\pi$. This implies that while the nanotriangles can show a CEP response at every harmonic of $f_{\mathrm{CEO}}$, the nanorods show a response only at even harmonics of $f_{\mathrm{CEO}}$. Therefore, the field-direction sensitivity of strong-field photoemission inhibits the CEP-dependent response of the nanorods at $f_{\mathrm{CEO}}$, just as we observe.

To further confirm our findings, we repeat the experiments outlined in Fig. 2 for seven other differently sized nanotriangle emitters and five other differently sized nanorod emitters. Specifically, we measure $I_{\mathrm{E}}$ as a function of $E_{\mathrm{p}}$ for each nanoparticle emitter type (see Supplementary Section IV for the measurements and extracted field enhancements); we measure the $f_{\text {CEO }}$ response from each nanoparticle type; and we measure the phase-stepping response of the $f_{\mathrm{CEO}^{-}}$ sensitive signals (see Supplementary Section V for further details). 

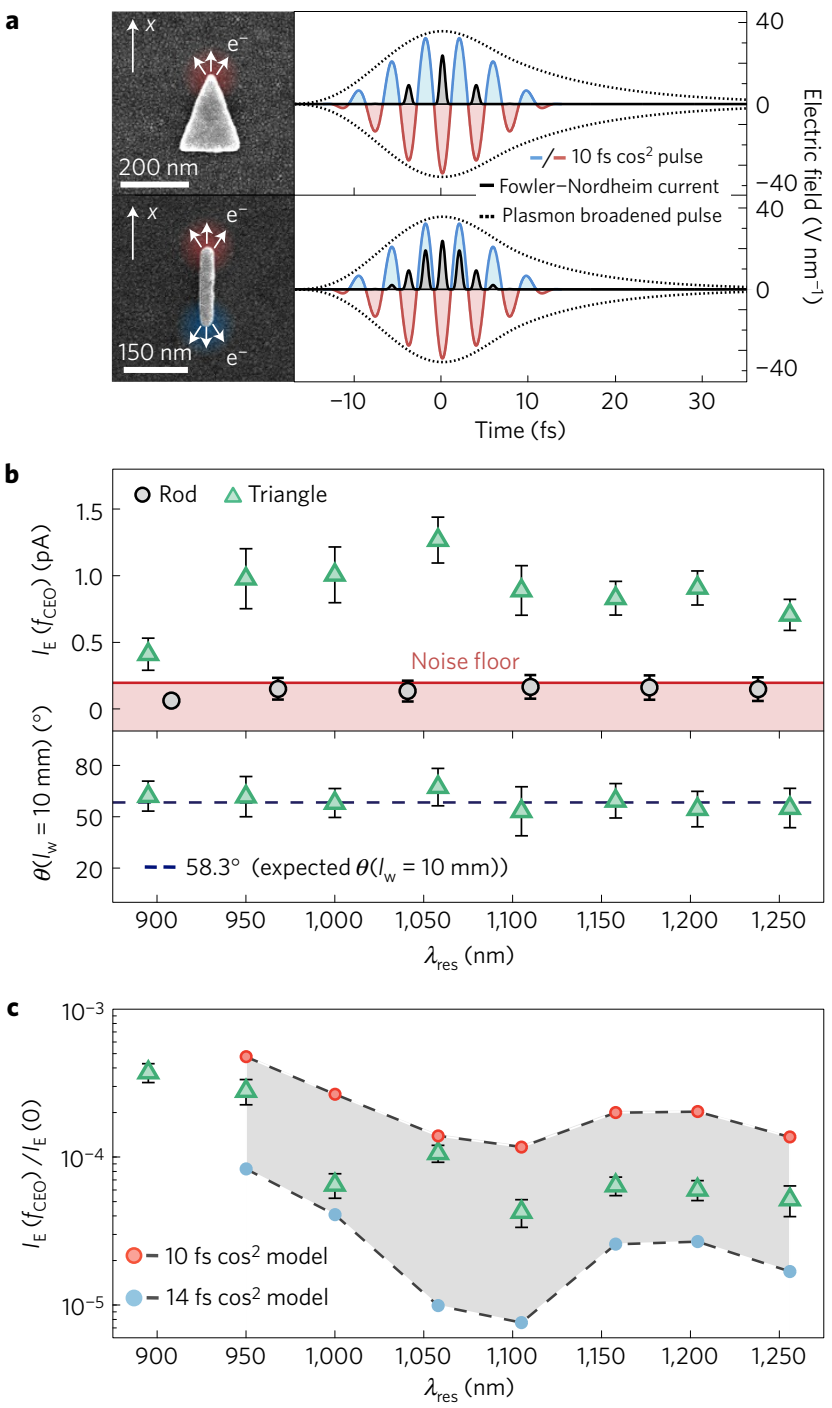

Figure 3 | CEP behaviours. a, Field-direction-sensitive emission: the nanotriangles have significant enhancement only at their apex, so they emit only from the red half-cycles of the sketched pulse (10 fs, $40 \mathrm{~V} \mathrm{~nm}^{-1}$ peak field, and $\cos ^{2}$ shape); the nanorods emit from both red and blue half-cycles. The resulting quasi-static Fowler-Nordheim emission (with arbitrary normalization) is overlaid in black. The dashed envelopes show sketches of the estimated plasmon-broadened near-field pulse envelopes (estimated via filtering with the oscillator fit to the nanoparticles' resonant behaviour). $\mathbf{b}, C E P$-sensitive current $I_{E}\left(f=f_{C E O}\right)$, that is, the amplitude of the first $f_{C E O}$ harmonic, for nanotriangles (green triangles) and nanorods (black circles) of differing resonant wavelengths. The noise floor is estimated as an average of the noise levels in each measurement. In the bottom panel, we display the results of the phase-stepping measurements on each nanotriangle; we show the fit value: $\theta\left(I_{\mathrm{w}}=10 \mathrm{~mm}\right) \pm \Delta \theta_{\text {r.m.s. }}$. c, The experimentally measured CEP sensitivity (defined here as $\left.I_{\mathrm{E}}\left(f=f_{\mathrm{CEO}}\right) / \mathrm{I}_{\mathrm{E}}(f=0)\right)$ and the predicted sensitivity from the quasi-static tunnelling model in equation (1). Each data point in $\mathbf{b}$ and $\mathbf{c}$ follows from minute-long measurements of the CEP-sensitive signal for the investigated nanoparticle emitter array, and the error bars follow from r.m.s. deviations of these measurements (see Supplementary Section $\vee$ for further details).

The results (plotted versus the resonant wavelength of the tested nanoparticle arrays) are displayed in Fig. $3 \mathrm{~b}$. We do not see an $f_{\text {CEO }}$ response for any of the five nanorod arrays while we see strong $f_{\text {CEO }}$ signals for all seven of the nanotriangle arrays. In the bottom panel of Fig. 3b, we display the results of the phase-stepping experiments on each nanotriangle emitter array.
Next, we compare the predictions of the quasi-static tunnelling model (equation (1)) to the experimental data. To estimate the near-field $F_{\text {np }}(t, \varphi)$ in equation (1), we start with our $\cos ^{2}$-shaped model laser pulses and filter them with the localized surface plasmon resonances; specifically, we filter them with the oscillator model fits to these resonances ${ }^{32}$. The resulting plasmon-broadened fields are then normalized to the incident peak field and multiplied by the experimentally retrieved field enhancement factors. Inserting these estimates for $F_{\text {np }}(t, \varphi)$ into equation (1) and integrating over the focused spot area, we arrive at an estimate for the total emission current as a function of $\varphi$. Further details on the model are included in the Supplementary Section VI.

In Fig. $3 c$ we compare the results of our model to the experiment. For comparison, we look at the CEP 'sensitivity', which we define as $I_{\mathrm{E}}\left(f=f_{\mathrm{CEO}}\right) / I_{\mathrm{E}}(f=0)$, that is, as the current in the $f_{\mathrm{CEO}^{-}}$ sensitive signal divided by the average current. The orange and blue data points in Fig. $3 \mathrm{c}$ are the predicted CEP sensitivities for $10 \mathrm{fs}$ and $14 \mathrm{fs} \cos ^{2}$ pulses respectively (the $14 \mathrm{fs}$ pulse corresponds to adding one additional optical cycle to the measured pulse duration). The sensitivity trends downwards as the resonant wavelength of the nanotriangles increases, that is, as the overlap of the plasmon resonance with the source spectrum (1-1.4 $\mu \mathrm{m}$ wavelength) increases. This trend is expected: when the resonance overlaps with the source spectrum, its filtering effects are the strongest, and $F_{\text {np }}(t, \varphi)$ is broadened relative to the incident field. Overall, from Fig. $3 c$, we see that the quasi-static tunnelling model gives reasonable predictions for the experimentally recorded sensitivity despite neglecting electron re-scattering effects, non-adiabatic emission, and detailed structure in the temporal and spatial profiles of the enhanced field.

As a last remark, we consider the application of our devices as CEP-sensitive photodetectors. The idea of using strong-field photoemission for CEP detection dates back two decades ${ }^{33}$, and recently, researchers have demonstrated chip-scale CEP detectors leveraging related physics ${ }^{34}$. To stabilize the $f_{\text {CEO }}$ of a laser oscillator, a SNR of $\sim 30 \mathrm{~dB}(\mathrm{RBW}>30 \mathrm{kHz})$ is typically required. Although falling short of this mark, our SNR could be dramatically improved by using a stereo collector arrangement. Currently, our detection electronics are limited by a large offset current. Arranging collector electrodes in a stereo, or balanced, configuration, for example, placing oppositely facing nanotriangle emitters on either side of a gap, we could eliminate this offset and significantly boost the SNR. Such detectors might allow direct $f_{\text {CEO }}$ stabilization and provide information about the absolute phase of the incident laser pulses.

Data availability. The data that support the plots within this paper and other findings of this study are available from the corresponding author on request.

Received 15 July 2016; accepted 9 November 2016; published online 19 December 2016

\section{References}

1. Corkum, P. B. Plasma perspective on strong-field multiphoton ionization. Phys. Rev. Lett. 71, 1994-1997 (1993).

2. Krausz, F. \& Ivanov, M. Attosecond physics. Rev. Mod. Phys. 81, 163-234 (2009).

3. Bormann, R., Gulde, M., Weismann, A., Yalunin, S. V. \& Ropers, C. Tip-enhanced strong-field photoemission. Phys. Rev. Lett. 105, 147601 (2010).

4. Schenk, M., Krüger, M. \& Hommelhoff, P. Strong-field above-threshold photoemission from sharp metal tips. Phys. Rev. Lett. 105, 257601 (2010).

5. Krüger, M., Schenk, M. \& Hommelhoff, P. Attosecond control of electrons emitted from a nanoscale metal tip. Nature 475, 78-81 (2011).

6. Yalunin, S. V., Gulde, M. \& Ropers, C. Strong-field photoemission from surfaces: theoretical approaches. Phys. Rev. B 84, 195426 (2011).

7. Herink, G., Solli, D. R., Gulde, M. \& Ropers, C. Field-driven photoemission from nanostructures quenches the quiver motion. Nature 483, 190-193 (2012). 
8. Krüger, M., Schenk, M., Förster, M. \& Hommelhoff, P. Attosecond physics in photoemission from a metal nanotip. J. Phys. B: At. Mol. Opt. Phys. 45, 074006 (2012).

9. Schertz, F., Schmelzeisen, M., Kreiter, M., Elmers, H.-J. \& Schönhense, G. Field emission of electrons generated by the near field of strongly coupled plasmons. Phys. Rev. Lett. 108, 237602 (2012).

10. Keathley, P. D. et al. Strong-field photoemission from silicon field emitter arrays. Ann. Phys. 525, 144-150 (2013).

11. Park, D. J. et al. Strong field acceleration and steering of ultrafast electron pulses from a sharp metallic nanotip. Phys. Rev. Lett. 109, 244803 (2012).

12. Dombi, P. et al. Ultrafast strong-field photoemission from plasmonic nanoparticles. Nano Lett. 13, 674-678 (2013).

13. Nagel, P. M. et al. Surface plasmon assisted electron acceleration in photoemission from gold nanopillars. Chem. Phys. 414, 106-111 (2013).

14. Piglosiewicz, B. et al. Carrier-envelope phase effects on the strong-field photoemission of electrons from metallic nanostructures. Nat. Photon. 8, 37-42 (2014).

15. Swanwick, M. E. et al. Nanostructured ultrafast silicon-tip optical field-emitter arrays. Nano Lett. 14, 5035-5043 (2014).

16. Hobbs, R. G. et al. High-density Au nanorod optical field-emitter arrays. Nanotechnology 25, 465304 (2014).

17. Hobbs, R. G. et al. High-yield, ultrafast, surface plasmon-enhanced, Au nanorod optical field electron emitter arrays. ACS Nano 8, 11474-11482 (2014).

18. Kusa, F., Echternkamp, K. E., Herink, G., Ropers, C. \& Ashihara, S. Optical field emission from resonant gold nanorods driven by femtosecond mid-infrared pulses. AIP Advances 5, 077138 (2015)

19. Keldysh, L. V. Ionization in the field of a strong electromagnetic wave. Sov. Phys. JETP 20, 1307-1314 (1965).

20. Büttiker, M. \& Landauer, R. Traversal time for tunneling. Phys. Rev. Lett. 49, 1739-1742 (1982).

21. Martin, Y. C., Hamann, H. F. \& Wickramasinghe, H. K. Strength of the electric field in apertureless near-field optical microscopy. J. Appl. Phys. 89, 5774-5778 (2001).

22. Arbouet, A., Houdellier, F., Marty, R. \& Girard, C. Interaction of an ultrashort optical pulse with a metallic nanotip: a Green dyadic approach. J. Appl. Phys. 112, 053103 (2012)

23. Giannini, V., Fernández-Domínguez, A. I., Heck, S. C. \& Maier, S. A. Plasmonic nanoantennas: fundamentals and their use in controlling the radiative properties of nanoemitters. Chem. Rev. 111, 3888-3912 (2011).

24. Biagioni, P., Huang, J.-S. \& Hecht, B. Nanoantennas for visible and infrared radiation. Rep. Prog. Phys. 75, 024402 (2012).

25. Wong, T. K. S. \& Ingram, S. G. Observational of Fowler-Nordheim tunnelling at atmospheric pressure using Au/Ti lateral tunnel diodes. J. Phys. D: Appl. Phys. 26, 979-985 (1993).

26. Driskill-Smith, A. A. G., Hasko, D. G. \& Ahmed, H. Nanoscale field emission structures for ultra-low voltage operation at atmospheric pressure. Appl. Phys. Lett. 71, 3159-3161 (1997).
27. Brimley, S., Miller, M. S. \& Hagmann, M. J. Field emission in air and space-charge-limited currents from iridium-iridium oxide tips with gaps below 100 nm. J. Appl. Phys. 109, 094510 (2011).

28. Han, J.-W., Oh, J. S. \& Meyyappan, M. Vacuum nanoelectronics: back to the future?-Gate-insulated nanoscale vacuum channel transistor. Appl. Phys. Lett. 100, 213505 (2012).

29. Dogariu, A., Shneider, M. N. \& Miles, R. B. Versatile radar measurement of the electron loss rate in air. Appl. Phys. Lett. 103, 224102 (2013).

30. Murphy, E. L. \& Good, R. H. Jr Thermionic emission, field emission, and the transition region. Phys. Rev. 102, 1464-1473 (1956).

31. Plech, A., Kotaidis, V., Lorenc, M. \& Boneberg, J. Femtosecond laser near-field ablation from gold nanoparticles. Nat. Phys. 2, 44-47 (2006).

32. Lamprecht, B., Krenn, J. R., Leitner, A. \& Aussenegg, F. R. Resonant and off-resonant light-driven plasmons in metal nanoparticles studied by femtosecond-resolution third-harmonic generation. Phys. Rev. Lett. 83, 4421-4424 (1999).

33. Xu, L. et al. Route to phase control of ultrashort light pulses. Opt. Lett. 21, 2008-2010 (1996)

34. Paasch-Colberg, T. et al. Solid-state light-phase detector. Nat. Photon. 8, 214-218 (2014).

\section{Acknowledgements}

We thank O. D. Mücke for helpful comments and J. Daley for assistance in device fabrication. This work was supported by the United States Air Force Office of Scientific Research (AFOSR) through grant FA9550-12-1-0499, the Center for Free-Electron Laser Science at DESY, and The Hamburg Center for Ultrafast Imaging: Structure, Dynamics and Control of Matter at the Atomic Scale, an excellence cluster of the Deutsche Forschungsgemeinschaft. W.P.P. acknowledges earlier support from an NSF graduate research fellowship; P.D.K. acknowledges support from an NDSEG graduate fellowship; and R.G.H. acknowledges support for the device fabrication work from the Center for Excitonics, an Energy Frontier Research Center funded by the US Department of Energy, Office of Science, Office of Basic Energy Sciences under award number DE-SC0001088. We thank A. Chu and J. Wanapun for additional support.

\section{Author contributions}

W.P.P. and F.X.K. conceived of the experimental concept. W.P.P. and R.G.H. fabricated the devices. W.P.P. constructed the laser source and experimental apparatus and carried out the measurements. W.P.P. developed the model, and W.P.P., R.G.H., P.D.K., K.K.B. and F.X.K. interpreted the results and composed the manuscript.

\section{Additional information}

Supplementary information is available in the online version of the paper. Reprints and permissions information is available online at www.nature.com/reprints. Correspondence and requests for materials should be addressed to W.P.P.

\section{Competing financial interests}

The authors declare no competing financial interests. 